\title{
X-linked Charcot-Marie-Tooth disease case with a novel missense mutation in GJB1 gene
}

\author{
Jong-Mok Lee ${ }^{1}$ and Jin-Hong Shin ${ }^{2 * *}$ \\ ${ }^{1}$ Department of Neurology, Kyungpook National University Hospital, Daegu, Korea \\ ${ }^{2}$ Department of Neurology, Pusan National University Yangsan Hospital, Yangsan, Korea
}

\begin{abstract}
X-linked Charcot-Marie-Tooth disease type 1 (CMTX1) is caused by the mutation in GJB1 gene, characterized by the transient central nervous system involvement and long standing peripheral polyneuropathy which does not fulfill the criteria of demyelination or axonopathy. We describe a 37-year-old man with progressive bilateral leg weakness since his early teen. He suffered transient right hemiparesis, followed by quadriparesis at 14 years of age. When we examined him at 37 years of age, he presented a distal muscle weakness on lower extremities with a sensory symptom. The nerve conduction study demonstrated a motor conduction velocity between 26 and $49 \mathrm{~m} / \mathrm{s}$. The whole exome sequencing revealed a novel variant c.136 G>A in GJB1. This report will raise awareness in this rare disease, which is frequently misdiagnosed early in its course.
\end{abstract}

Key words: Charcot-Marie-Tooth disease, Connexins, Central nervous system.

\section{Introduction}

X-linked Charcot-Marie-Tooth disease (CMTX) is the second most common among all types of Charcot-Marie-Tooth disease (CMT). Its prevalence is estimated to be $7 \%$ to $16 \%$ of all CMT cases, while it is a rare cause of peripheral neuropathy [1]. Most of CMTX cases are by mutations in GJB1 gene, and are labeled as CMTX1 (MIM \#302800). CMTX1 share common features with CMT such as progressive motor weakness, wasting of the lower extremities and foot deformities, hypoactive deep tendon reflexes (DTRs), and sensory abnormalities. Interestingly, CMTX1 has distinct features not found in most other types of CMT: the antecedent central nervous system (CNS) involvement, which involves white matter changes in magnetic resonance imaging (MRI) and magnetic resonance spectroscopy abnormalities [13]. CMTX1 follows $X$-linked dominant inheritance, and male patients are more severely affected than female patients.

$G J B 1$ encodes a gap junction protein, also known as connexin 32. Mutation in $G J B 1$ can lead to dysfunction of ion exchange in the cell membrane. So far, more than 400 mutations in GJB1 have been identified [1]. Here, we report a CMTX1 case with a novel mutation in $G J B 1$. We believe this report may expand the mutation spectrum CMTX1, and help raise awareness of CMTX1 often misdiagnosed as CNS disease.

\section{Case}

A 37-year-old man visited Kyungpook National University Hospital presenting slowly progressive bilateral leg weakness, preferentially affecting distal leg, since his early teen. At his age of fourteen years, he experienced transient dysarthria, right central facial palsy, and hemiparesis with tingling sensation follow-

Received: 14 June 2018, Revised: 2 August 2018, Accepted: 3 August 2018, Published: 31 December 2018

${ }^{*}$ Corresponding author: Jin-Hong Shin, M.D., Ph.D. (iD http://orcid.org/0000-0002-5174-286X

Department of Neurology, Pusan National University Yangsan Hospital, 20 Geumo-ro, Mulgeum-eup, Yangsan 50612, Korea.

Tel: +82-55-360-2122, Fax: +82-55-360-2521, E-mail: shinzh@gmail.com

Conflict of interest: The authors declare that they do not have any conflicts of interest.

(C) This is an open-access article distributed under the terms of the Creative Commons Attribution Non-Commercial License (http://creativecommons.org/licenses/by-nc/4.0/) which permits unrestricted non-commercial use, distribution, and reproduction in any medium, provided the original work is properly cited.

(c) Copyright 2018 by the Korean Society of Medical Genetics and Genomics 
ing upper respiratory infection with fever. These symptoms lasted 3 hours. On the next day, the same symptom recurred. Muscle weakness of right arm and leg corresponded to 4 by Medical Research Council (MRC) muscle power grading system. The weakness progressed to 2 on the right and 4 on the left limbs one day after hospitalization. And dysarthria and ataxia on right limbs were identified. The brain MRI showed fluid-attenuated inversion recovery (FLAIR)-high signal changes in the white matter of parieto-occipital area and splenium (Figs. 1A and B). These symptoms were treated with intravenous methylprednisolone under diagnosis of acute disseminated encephalomyelitis and recovered completely in five days. Since then, he had suffered intermittent headache without an objective neurological deficit and remained undiagnosed until his age of 37 years. Although his foot drop had made gait difficulty, he could walk by himself. Sensory deficit was noted in his lower legs. Two male cousins on his mother's side also had foot drops and further information could not be obtained.

A neurological examination at 37 years of age revealed weakness in ankle dorsiflexion and great toe extension bilaterally cor-
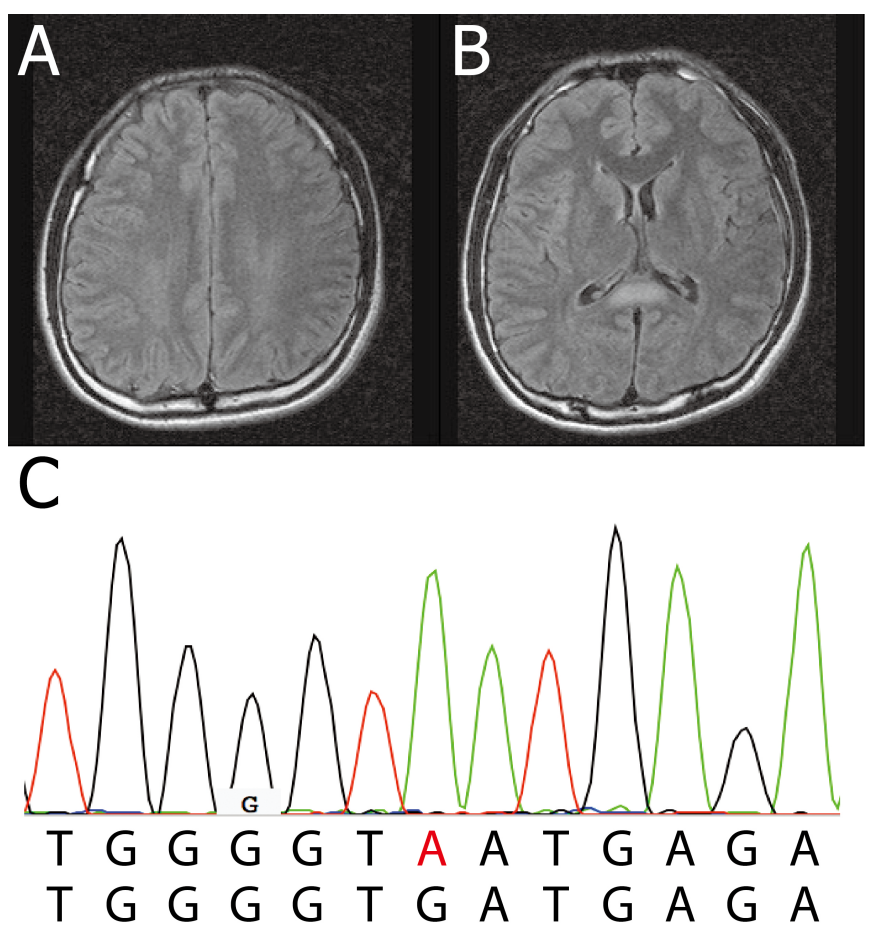

Fig. 1. Brain magnetic resonance image (MRI) and chromatogram by the Sanger sequence. The brain MRI at 14 years of age shows fluidattenuated inversion recovery-high signal changes in the white matter of parieto-occipital area and splenium (A and B). The chromatogram of Sanger sequence reveals that nucleoside substitution from guanine to adenine at the position of 136 base in $G J B 1$ gene (c.136G $>A$, p.Asp46Asn, NM_000166.5) (C). responding to 4 by MRC grading system. The muscle strengths of upper extremities and proximal lower extremities were normal. The DTRs were all reduced. Pes cavus with hammer toes was evident on both feet.

The nerve conduction studies demonstrated low amplitude sensorimotor polyneuropathy with sensory conduction velocities ranging 31 to $45 \mathrm{~m} / \mathrm{s}$ and motor conduction velocities ranging 26 to $49 \mathrm{~m} / \mathrm{s}$. Brainstem auditory evoked potential revealed a prolonged interpeak latency between wave I and III, rendering the conduction defect between acoustic nerve and lower brain stem.

The routine laboratory tests, chest roentgenogram, and electrocardiogram were all normal. According to the findings of history, examination, and laboratory, this patient was graded as 10 by the CMT neuropathy score (CMTNS) [4].

Based on the clinical information, we skipped the gene test for PMP22 duplication and proceeded directly to the whole exome sequencing. Variants affecting protein structure or function, including non-synonymous variants, frameshifts, or variants affecting splicing, were investigated. The variants showed a depth of more than 30 and were filtered by allele frequency of PopFreqMax less than 0.0001, which consists of the Genome Aggregation Database, Exome Aggregation Consortium (ExAC), and 1000 Genome (1000 genome). In the case of point mutation, we evaluated the impact of mutations using SIFT/PROVEAN and Polyphen-2 systems (Supplementary Table 1). A novel missense mutation c.136G >A (p.Asp46Asn, NM_000166.5, Fig. 1C) in $G J B 1$ was detected, which is not present in ExAC, the 1000 genome, Human Gene Mutation Database or Inherited Peripheral Neuropathies Mutation Database (http://www.molgen.ua.ac. be/CMTMutations/). SIFT, PROVEAN, and PolyPhen-2 systems predicted that it would be "deleterious" with a score of -4.81 , "damaging" with a score of 0.001 , and "damaging" with a score of 1 , respectively.

\section{Discussion}

We could recognize CMTX 1 based on the symptoms: mild phenotype, maternal cousins with the same symptom, peripheral polyneuropathy not exactly either demyelinating or axonal features, and previous episodes of CNS involvement. The diagnosis was confirmed by detecting a novel mutation in $G J B 1$ c.136G $>A$ (p.Asp46Asn), which is predicted to be pathogenic by in silico test. Moreover, different amino acid substitution at the same position (c.137A $>$ G, p.Asp46Gly) had been reported to be pathogenic in $G J B 1$ [5]. 
The fever, one of the precipitating factors affecting central involvement [2], had also been noticed in this patient. And the decreased number of functioning gap junctions between oligodendrocytes and astrocytes leading to disturbed gap junction communication and intercellular fluid exchange of ions and small molecules was believed to be also precipitated by the exposure to high altitude, exercise, concussion, singing, and hyperventilation [2].

One of the recent reports introduced clinical characterization of Korean patients with CMTX1 [6]. In this study, mean age of onset and CMTNS in symptomatic male patients was reported to be 18.4 and 13.2, respectively [6]. And severe phenotypical presentation was suggested in the patient with mutations located in extracellular (EC) domain [6]. The clinical presentation of our patient including an age of onset and CMTNS is consistent with the previous study, and motor velocities of the ulnar nerves are slower than those of the median nerves in our patient, which supports the previous study [6]. However, our patient seems to show less severe phenotype, even the mutation c. 136G >A is located in EC domain.

In terms of the genotype-phenotype correlation concerning basic functional studies, reduced GJB1 expression and intracellular localization leading to altered gap junction function in cell model has been suggested to have a positive correlation with more severe phenotypes [3]. Complete loss of gap junction $\mathrm{Ca}^{2+}$ permeability and cytosolic expression of GJB1 appeared to be associated with an earlier disease onset and more severe phenotype (mean age of onset, 19 years old; mean CMTNS, 13.4), whereas preservation of gap junction permeability and predominant cell membrane expression of GJB1 tended to have a later onset and milder phenotype (mean age of onset, 35.8; mean CMTNS, 8.8) [3]. Moreover, phenotypes owing to nonsense or frameshift mutations appear to be more severe than those of missense mutations [3,7].

We reported a novel mutation in $G J B 1$ associated with juvenileonset CMTX1. In addition to the peripheral neuropathy, reversible
CNS involvement affecting corpus callosum and periventricular area was noted on MRI. Our patient has been undiagnosed for a long time, which is most likely due to the low level of awareness for CMTX1. Therefore, CMTX1 should be considered when encountering the patient with CNS involvement, hearing impairment, and foot drop. And clinical awareness of CMTX 1 will lead to the successful diagnosis of more cases aided by next generation sequencing technology.

\section{Supplementary Material}

Supplementary table can be found via https://doi.org/10.5734/ JGM.2018.15.2.107

\section{References}

1. Wang $Y$, Yin F. A review of $X$-linked Charcot-Marie-Tooth disease. J Child Neurol 2016;31:761-72.

2. Kleopa KA, Abrams CK, Scherer SS. How do mutations in GJB1 cause X-linked Charcot-Marie-Tooth disease? Brain Res 2012;1487:198205.

3. Tsai PC, Yang DM, Liao YC, Chiu TY, Kuo HC, Su YP, et al. Clinical and biophysical characterization of 19 GJB1 mutations. Ann Clin Transl Neurol 2016;3:854-65.

4. Murphy SM, Herrmann DN, McDermott MP, Scherer SS, Shy ME, ReilIy MM, et al. Reliability of the CMT neuropathy score (second version) in Charcot-Marie-Tooth disease. J Peripher Nerv Syst 2011;16:191-8.

5. Park HK, Kim BJ, Sung DH, Ki CS, Kim JW. Mutation analysis of the PMP22, MPZ, EGR2, LITAF, and GJB1 genes in Korean patients with Charcot-Marie-Tooth neuropathy type 1. Clin Genet 2006;70:253-6.

6. Hong YB, Park JM, Yu JS, Yoo DH, Nam DE, Park HJ, et al. Clinical characterization and genetic analysis of Korean patients with $\mathrm{X}$ linked Charcot-Marie-Tooth disease type 1. J Peripher Nerv Syst 2017;22:172-81.

7. Chen SD, Li ZX, Guan YT, Zhou XJ, Jiang JM, Hao Y. A novel mutation of gap junction protein $\beta 1$ gene in $X$-linked Charcot-Marie-Tooth disease. Muscle Nerve 2011;43:887-92. 